\title{
Intelligent Home Environment using Blue Eyes Technology
}

\author{
Sreekanth Sreekumar \\ Dept. of Computer Science and Engineering \\ College of Engineering, Trivandrum
}

\begin{abstract}
Intelligence is the catchword of the $21^{\text {st }}$ century. More and more intelligent devices are produced which reduces human effort and such devices are now taught to think for itself. "Blue eyes" is a technology doing the same. It gives computational systems emotional and perceptual capabilities which help them to improve their interaction with humans. Thus machines become more human like and make intelligent decisions by themselves. 'Blue Eyes' has many practical applications, and this paper implements a technique by which the technology can be applied to a home. A home modified in this way can sense the emotions of its inhabitants and respond in the most appropriate way. This produces a stress free and peaceful atmosphere at home, which can be a big relief after a hectic day at work.
\end{abstract}

\section{General Terms}

Emotion Detection, Pattern Recognition, Facial Detection, Pressure Detection, Automation, Intelligent.

\section{Keywords}

Blue eyes, Image Processing, Facial Emotion Detection, Pressure Sensing, Home Automation.

\section{INTRODUCTION}

Blue Eyes is a technology conducted by the research team of IBM at its Almaden Research Centre (ARC) in San Jose, California since 1997. Blue eyes technology makes a computer to understand and sense human feelings and behavior and also enables the computer to react according to the sensed emotional levels. The aim of the blue eyes technology is to give human power or abilities to a computer, so that the machine can naturally interact with human beings as we interact with each other. The process of making a computer having sensing and emotional capabilities is known as "Affective Computing". The steps involved for designing such type of computers are given below.

1. Process of giving sensing capacity.

2. Human Emotion Detection or Affect Detection.

3. Respond appropriately and properly.

Section 2 discusses the basic techniques used to implement blue eyes computational systems. Section 3 deals with how these techniques can be extended in home automation and section 4 provides the conclusion of this paper. Section 5 provides future expansion possibilities in this topic.

\section{BASIC IMPLEMENTATION}

\subsection{Emotional Mouse}

It obtains physiological data and emotional state such as heart beat, pressure, temperature etc. through the touch of user on mouse where different sensors (such as pressure sensor, heart beat sensor, GSR sensor, temperature sensor) are deployed inside it. Then it determines the emotional situation of the user. Using emotional mouse we understand the mood variations and psychological state of the user with a simple touch of the mouse.

\subsection{Manual and gauge input cascading (MAGIC pointing)}

A webcam is used to quickly determine the glints and pupils of the user under variable and realistic lightning conditions and wrap the cursor to every new object, the user looks at. The cursor is wrapped around an area or boundary within which the target object exists with $95 \%$ probability. This reduces the length at which the cursor should be moved manually in order to reach the target object.

\subsection{Artificial intelligent speech recognition}

For implementing the Artificial Intelligent Speech Recognition system in Blue Eyes technology, the working environment should be very important. It uses a concept called Natural Language Processing (NLP). The main goal of the Natural language processing (NLP) is to understand the users input and react according to these inputs. The input data or words are continuously scanned and finds matches against inside stored known data or words. And after identifying the key words, the corresponding actions are carried out by the machine.

\subsection{Simple user interest tracker (SUITOR)}

The SUITOR continuously analyses the user that where his eye focus on the personal computer screen. The SUITOR has the ability to determining the topic of interest of the user and also according to this it can able to deliver the appropriate data to a handheld device. E.g.: If you are reading a headline, the story pops up in the browser window in the background.

\section{INTELLIGENT AUTOMATION}

Human emotion is a visible manifestation of effective state, cognitive activity, emotional state and personality. A human emotion is easily captured through his facial expressions and the pressure by which he handles objects. This paper tries to capture the psychological state of a person from the moment he enters the home. In response to these detected emotions the home automation central system responds accordingly. The techniques provided will ensure a prompt action, which can soothe the person and act towards the betterment of his mood.

\subsection{Architecture}

Like traditional blue eyes technology this proposed model has

1) Data Acquisition Unit and a

2) Central System Unit 
The data acquisition unit gathers date received from GUI equipment's around the house and the central system unit processes this information and decides on the response actions. In this paper, we look at the possibility of linking the two modules under the Wi-Fi connection system of the home. The Wi-Fi module provides a wireless interface between the Central System Unit (CSU) and the GUI sensors.

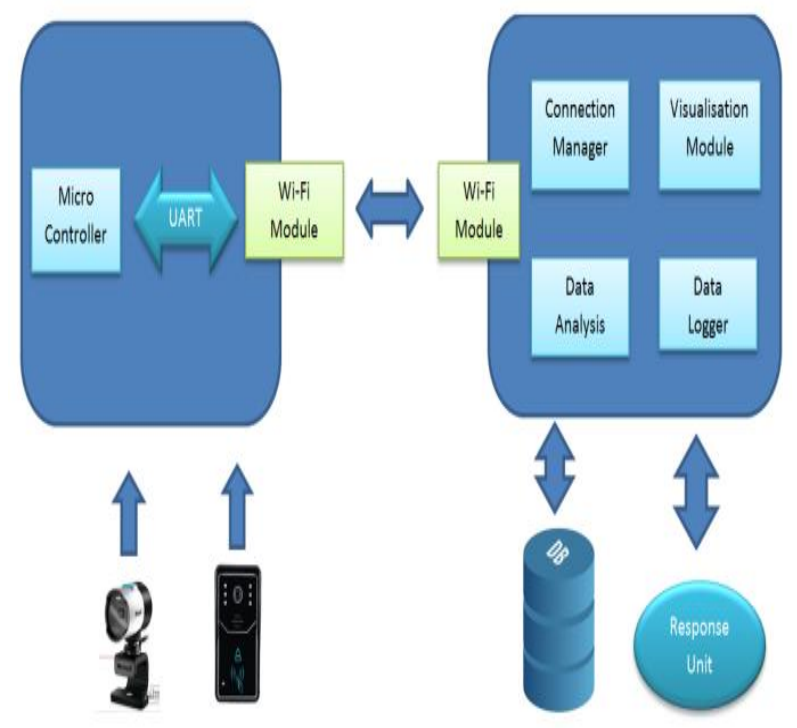

Fig1: The system overview

\subsection{Detecting facial expression of an individual}

In this paper the technique proposed in [1], which deals with the detection of emotions of human through the texture of eye because eyes are "window to the soul" that they can tell much about person internal state just by gazing into them, a camera will capture the image of a person and focuses on the eye area by using texture filtering algorithm which is then compared with the list of images stores in data base. The best image that identifies the emotion of a person is shown on the window. This camera when aimed at the main doorway can detect the emotion of a person from the moment he enters the house.

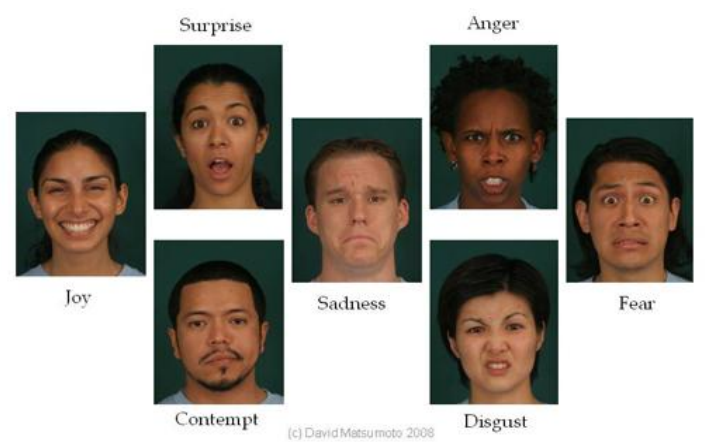

Fig3: Showing variation in facial gestures according to psychological situation.

The procedure follows the below flowchart,
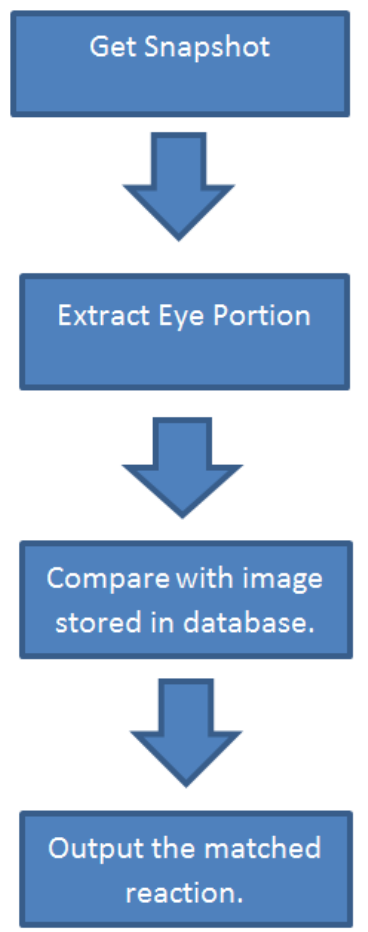

Fig2: Flowchart showing stages of emotion detection.

Thus once an idea about the emotion of the person is detected, a response which facilitates the improvement of his psychological condition can be activated. This can also be used for a variety of other purposes. This camera situated aiming at the doorway can also detect the presence of intruders. When faces which are not present in the trained database of the system are detected, the intelligent home alerts the presence of an intruder. It can also be used to make sure that children (under the age of 7) do not pass through the doorway without adult supervision. In such a way, parents can also ensure the safety of their children.

\subsection{Detecting pressure by which the user presses a switch}

A force-sensitive interface can be implemented by checking the flashlight source blocked by a human hand, 3 monitoring the reduction of sound volume by covering the microphone reception hole, 4 or estimating the damped motor vibration with accelerometers. But these techniques can only work in harmony with extra hardware. As proposed in [2], this paper looks at a solution used in touch phones called ForcePhone which detects the pressure at which a button is pressed in the mobile phone. We can determine a lot about a person's emotional state by the way in which he handles objects. An impatient person is sure to press the calling bell of his home hard and for longer duration. Implementing a counter hardware we can also predict his mood depending on the number of times he presses the electronic bell. From the pressure at which he/she presses the switch and from the number of times the switch is turned on, the central system is able to determine and respond timely to the person's mood. This paper discusses the application of ForcePhone for this procedure.

ForcePhone provides a force-sensitive input interface to the touchscreen. It estimates the user-applied force by using the structure-borne sound propagation-that is, the sound transmitted through subtle vibrations of the device body. 
Sound is a mechanical wave broadcasted by compressions and rarefactions. The most common material for sound to propagate is the air, which is known as airborne propagation, but when the sound is generated and received by the same device, its body becomes another pathway for the sound to travel. When force is applied to the device (switch), it restricts the device body's vibration with the sound, thus degrading the sound traveling through this structure-borne pathway. ForcePhone estimates the amount of force applied to the device by monitoring the degree of this degradation.

ForcePhone actively plays an inaudible sound with the device speaker and then picks up this sound with the device's microphone. This sensor data is then used to improve the force estimation and reduce the number of false detections. The design of sound is critical to system performance. Although there are many other possible options, ForcePhone's current design uses a 1,200-sample linear chirp from 18 to 24 $\mathrm{kHz}$. A hamming window is multiplied to the first and last of 300 samples to eliminate the audible noise caused by spectral leakage.

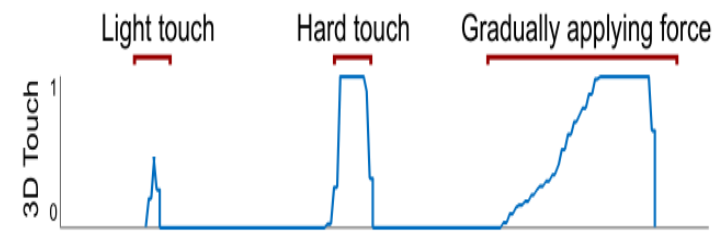

Fig4: [2] Responses to different touch.

In ForcePhone, these noises are identified and removed by time stamping the received audio signal. For example, the reflection from an object 10

$\mathrm{cm}$ away will be received 28 samples later than the airborne propagation, because it travels $10 \mathrm{~cm}$ farther. Moreover, sound usually travels 100 times faster in a solid phone body. 8 Thus, the structure-borne propagation will be received 21 samples ahead of the airborne propagation when the microphone and the speaker are $15 \mathrm{~cm}$ apart. Based on these observations, ForcePhone uses the signal that's 20 samples ahead of the airborne propagation as the indicator of the structure-borne propagation, thus removing the most undesirable noise. Note that the reference of airborne propagation is assumed to be the strongest audio correlation, because the sound energy decays faster through the solid phone body and is absorbed more on the reflection paths than air. Ideally, the signals detected before the airborne sound should represent only the structure-borne sound propagation. However, in measurement, the signals from multiple paths were mixed together due to the audio distortion and the adoption of windowing. The windowing process suppressed the frequency domain signal leakage but incurred the timedomain signal leakage. This 20-sample-ahead sampling heuristic thus includes both air- and structure borne propagations.

To get a reliable estimation of the applied force, ForcePhone uses the sound strength when the touch begins as a reference to estimate the subsequent change caused by the force applied later. That is, the signal components from the other paths are removed by subtracting the current estimation from the reference signal. This heuristic is designed based on the assumption that the signal of the other paths won't change as significantly as the change of structure borne signals when the user applies force to the phone.

\subsection{Appropriate response action}

The response action can be in the form of anything that progresses towards the improvement of the psychological state of the inhabitant. It can be the dimming of the lights to a particular level, it can be, and it can be the presence of an immediate telephonic call to a loved one. In this paper, the method proposed is to play a song or a chord that goes in harmony with the user.

One of the reasons why music is everywhere in our everyday life is that one of the fundamental functionality of music is triggering emotion. Most of the people empirically know that music triggers various emotions such as happiness, sadness, and joyfulness, to name a few. Different music genres can make or break your mood, we all know this. Music is important for regulating our emotional state, can impact our focus and has an effect on the overall way we feel. Music has been used for centuries to change energy levels, improve mood and even to help people who are ill or suffering from emotional trauma to heal. According to research published in The Journal of Positive Psychology, people can successfully improve their moods and boost their overall happiness in just two weeks, simply by listening to specific mood-boosting music. Researchers from McGill University in Montreal said the response to music comes from the chemical dopamine. Correlating increased levels of dopamine in the brain to corresponding music proves that humans obtain pleasure from listening to music.

The programmed script takes one or more audio clips from a database, wherein an emotion based algorithm has already separated songs according to the level of mental arousal they produce. An appropriate song from the list is choses and played across the house. The database can also be manipulated according to user preferences. When a user who has been detected as being sad, manually listens to a particular song often, then that song would be given higher priority when such situations arise again in the future. 


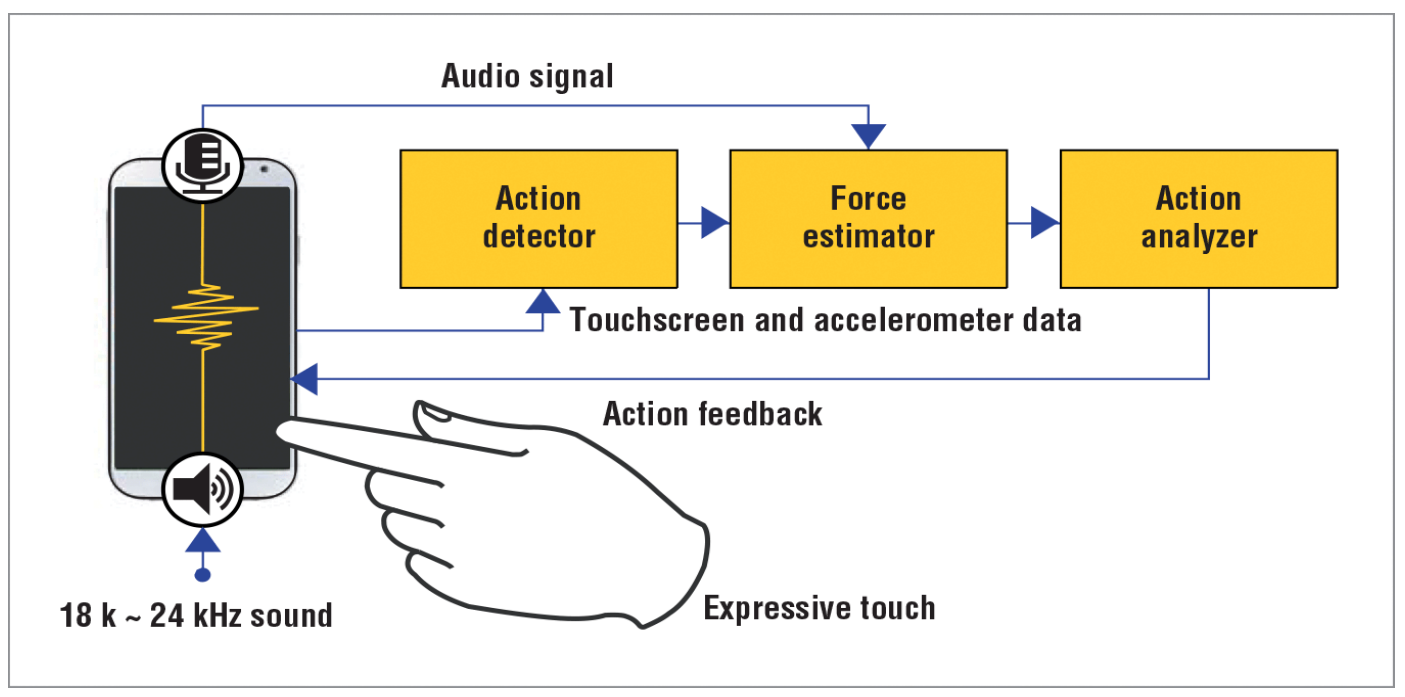

Fig 5: [2] Force applied to the phone damps the inaudible sound sent from the phone's speaker to its microphone. Accelerometer and gyroscope readings are used to avoid other audio signal noises caused by movements.

\section{CONCLUSION}

This paper proposes key ideas through which the emotional state of an individual can be detected and how a prompt action can help in the betterment of his mood. The easiest way by which his psychological state can be determined is by using the the fact that different eye colours and their intensity results in change in emotions. Another method the paper proposes is to find the pressure at which he handles objects around him, like the pressing of a switch. ForcePhone estimates the userapplied force by using the structure-borne sound propagation - that is, the sound transmitted through subtle vibrations of the device body. When force is applied to the device (switch), it restricts the device body's vibration with the sound, thus degrading the sound traveling through this structure-borne pathway. ForcePhone estimates the amount of force applied to the device by monitoring the degree of this degradation. Finally, a response action is taken by searching a database for the appropriate song. The database is classified in accordance with the arousal of dopamine in the body and through personal preferences of the inhabitants. The motive of this research is the providing of a stress free environment which increases productivity and health of all under it.

\section{FUTURE EXPANSIONS}

Several expansions can be done to this paper which involves its advent to common home appliances. Using tracking methods through eye gazing, techniques which reduce human effort such as, switching on an appliance as the user looks at it, energy efficient functioning of products, automatic dimming and brightening of lights can be implemented in a home environment. Detecting speech of a person inside the house, the central system can further extend its capabilities. An automatic vacuum system which identifies unpleasant odor and removes it can be another feature implemented. Likewise, a full-fledged automated home can be developed in recent years.

\section{ACKNOWLEDGMENTS}

I express my sincerest gratitude towards my research guide Prof. Vipin Vasu of Computer science department, College of engineering, Trivandrum for sharing his invaluable experience and guiding me throughout the completion of this paper. I thank the faculty and students of the same for their support. Lastly, I express my gratitude to the referenced entities for having inspired me in the making of this model.

\section{REFERENCES}

[1] Blue eyes technology, Mizna Rehman Mizna; Mamta Bachani; Sundas Memon, Eighth International Conference on Digital Information Management (ICDIM 2013).

[2] ForcePhone: Software Lets Smartphones Sense Touch Force, Yu-Chih Tung; Kang G. Shin, IEEE Pervasive Computing, Year: 2016

[3] M. Zentner, D. Grandjean, and K. Scherer, "Emotions evoked by the sound of music: Characterization, classification, and measurement," Emotion, vol. 8, no. 4, pp. 494-521, 2008Tavel, P. 2007 Modeling and Simulation Design. AK Peters Ltd.

[4] ]X. Hu and J. S. Downie, "Exploring mood metadata: relationships with genre, artist and usage metadata," Proc. ISMIR, pp. 67-72, Sept. 2007.

[5] S.Madhumitha,Slide Share, Blue Eyes Technology, March 2013, <www.slideshare.net/Colloquium/blue-eyestechnology $>$.

[6] W. O. Chase, How Music REALLY Works: The Essential Handbook for Songwriters, Performers, and Music Students,2nd ed. Vancouver, Canada: Roedy Black Publishing, 2006.

[7] S. Hwang and K.-y. Wohn, "Pseudobutton: Enabling Pressure-Sensitive Interaction by Repurposing Microphone on Mobile Device," Proc. Extended Abstracts on Human Factors in Computing Systems (CHI), 2012, pp. 1565- 1570. 